International Journal of Information Sciences and Techniques (IJIST) Vol.4, No.3, May 2014

\title{
TRACKING AND COUNTING THE VEHICLES IN NIGHT SCENES
}

\author{
S. Kanagamalliga ${ }^{1}$, Dr. S. Vasuki ${ }^{2}$, A. Kanimozhidevi ${ }^{3}$, S. Priyadharshni ${ }^{4}$, S. \\ Rajeswari $^{5}$ \\ ${ }^{1}$ Assistant Professor, ${ }^{2}$ Professor and Head, ${ }^{3,4,5}$ U.G Student \\ Department of Electronics and Communication Engineering, Velammal College of \\ Engineering and Technology, Madurai
}

\begin{abstract}
:
The main objective of traffic surveillance system is to reduce the risk caused by accident. Many papers published were concerned only about the vehicle detection during daytime. But we proposed a method to detect the vehicle during night time. The main objective of our paper is to count the vehicles in night time traffic scenes. It consists of three phases, they are vehicle's headlight segmentation, tracking and pairing. We know that in night time, only visible thing will be the headlight of the vehicle therefore we are counting the vehicle based on the headlight information. Initially the headlights of a vehicle is segmented based on the analysis of headlight size, location and area. Then tracked via a tracking procedure designed to detect vehicle and then headlights are paired using the connected component labeling. Based on the pairing result we can count the vehicles. Here we are using fuzzy hybrid information inference mechanism for error compensation. Here by using fuzzy logic we can generate the rules based on the size, color and position. In particular we are generating the rules based on the distance between the headlights of a vehicle.Thereby we can improve the accuracy while counting the vehicle.
\end{abstract}

\section{Keywords:}

Headlight pairing,headlight tracking, bidirectional algorithm, intelligent transportation system

\section{INTRODUCTION:}

Traffic surveillance system extracts accurate traffic information for traffic flow control such as vehicle count, vehicle classification and vehicle speed..Hence in the day time counting the vehicle will be somehow easier than in the night time because the vehicles will be visible in the day time whereas in the night time those vehicles will not be visible due to dim light and also that there will be a strong reflection on road surface due to vehicle's headlights. Hence it will be difficult to calculate the vehicles in the night time.And also it is noted that in night time only the headlight of the vehicle will have high intensity value.Therefore by that information we can detect the vehicles in night scenes.In our existing paper,they concentrated on night time vehicle headlight detection and tracking in gray scale image. During the tracking and pairing the headlight, bidirectional algorithm has been used.In particular they used a vanishing point calculation for tracking and pairing the vehicle's headlight.There is a problem in the vanishing

DOI : $10.5121 /$ ijist.2014.4320 
International Journal of Information Sciences and Techniques (IJIST) Vol.4, No.3, May 2014

point calculation due to this counting will not be an accurate one we will see about this in detail existing work description.. In our paper, we present a approach for vehicle headlight detection, tracking, and pairing in night time traffic surveillance video. Through a simple yet effective bidirectional reasoning algorithm by incorporating the size, position, edge based, and motion information. Finally, the trajectory of the vehicle's headlight is employed to calibrate the surveillance camera to differentiate moving vehicles from motionless background scenes based on change detection. Other studies [3][4] use spatial-temporal difference features to segment moving vehicles, while the methods in utilize techniques based on background subtraction to extract moving vehicles.

\section{EXISTING WORK DESCRPTION}

The headlights of the vehicle's are detected,tracked and paired in night night time by the existing work.It contains two features in headlight detection of the vehicle. They are reflection intensity map and suppressed map. The reflection intensity map is obtained by the light attenuation model and the reflection suppressed map is obtained by the laplacian of Gaussian filter.From this the reflections on the road surface can removed.Then the headlights are tracked and paired by using the bidirection reasoning algorithm. Through this the number of vehicles can be counted. Finally, vehicle's speed has estimated by using the surveillance camera.The bidirectional reasoning obtained by calculating the position,size, vanishing point and the motion information. The following tabulation represents the number of vehicles counted per frame by using the vanishing point calculation.

\begin{tabular}{|l|l|l|l|}
\hline S.NO & $\begin{array}{l}\text { NUMBER OF } \\
\text { VEHICLES }\end{array}$ & $\begin{array}{l}\text { DETECTION } \\
\text { TIME }\end{array}$ & ACCURACY \\
\hline 1 & 25 & 1.2944 & $92.5 \%$ \\
\hline 2 & 19 & 0.3419 & $93.4 \%$ \\
\hline 3 & 21 & 0.3230 & $94.5 \%$ \\
\hline 4 & 18 & 0.3012 & $95.9 \%$ \\
\hline
\end{tabular}

Fig(i) tabulation represents the number of vehicles detected per frame

\section{PROBLEM IN THE EXISITING WORK}

In this exisiting work vanishing point calculation has been used for pairing the headlights of the vehicle. There might be a problem occur if one of the headlights of the four wheeler is wrongly paired with the two wheeler headlight.At that time it may include that wrongly paired vehicle headlights also one of the count.The pairing of vehicle headlights by using the vanishing point calculation is shown in this figure(a).From this it is noted that the one of the headlights of the some cars are wrongly paired with the one of the headlights of the other cars.So the existing work will not give the correct result. 


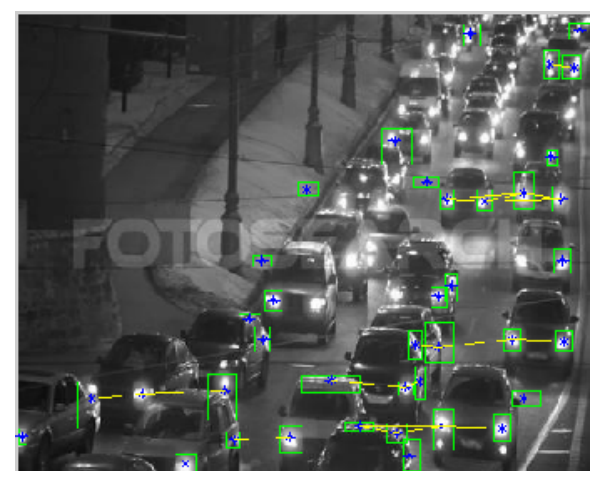

Fig(a) shows the pairing of vehicle's headlight using vanishing point calculation.

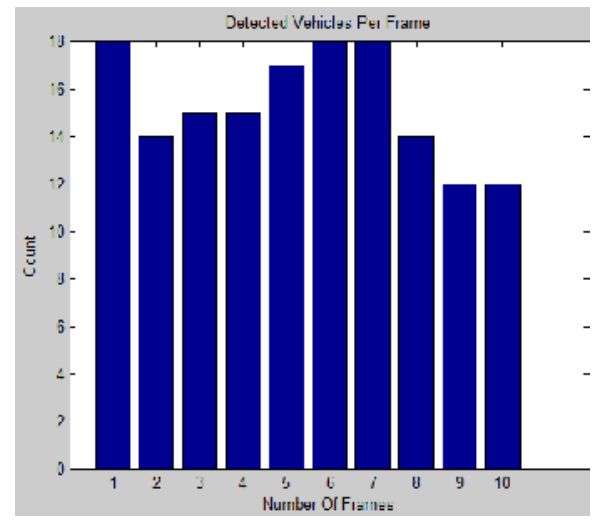

Fig(b ) graph has been plotted number of frames versus count

\section{PROJECT DESCRIPTION}

In our proposed we are counting the vehicles in night time in a traffic area. The proposed method has three phases. They are preprocessing, segmentation, tracking and pairing the vehicle's headlight. Intially the captured video should be converted into the frame and we subject this frames for preprocessing. By using the filters the noise has been removed from the frame. Here we are counting the vehicle in night time. Therefore in night time only vehicle's headlight will be visible.Hence with the help of headlight information only we can count the vehicles for that first we have to segment those vehicle's headlight. For segmenting the vehicle's headlight we are using the edge based technique. The main objective of segmentation is to eliminate the road reflection due to vehicle's headlight. Once the headlight has been segmented then those headlight have to be tracked and paired. For pairing the vehicle headlight we are using the bidirectional reasoning algorithm. for tracking the vehicle's headlight we are using the connected component labeling(CCL).Based on paired results we can count the vehicle in night scenes. And also it is noted for error compensation we using the fuzzy hybrid interference information mechanism(FHIIM).By using the fuzzy logic we can generate the rule. Rule can be generated based on size, shape, color, distance between two headlights in a vehicle. 
International Journal of Information Sciences and Techniques (IJIST) Vol.4, No.3, May 2014

In particular we are generating the rules based on the distance between the two headlights of a vehicle. Thereby we can improve the accuracy while counting the vehicle.

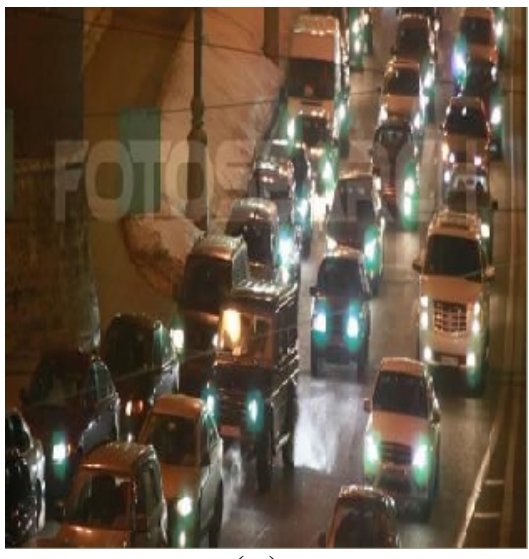

(c)

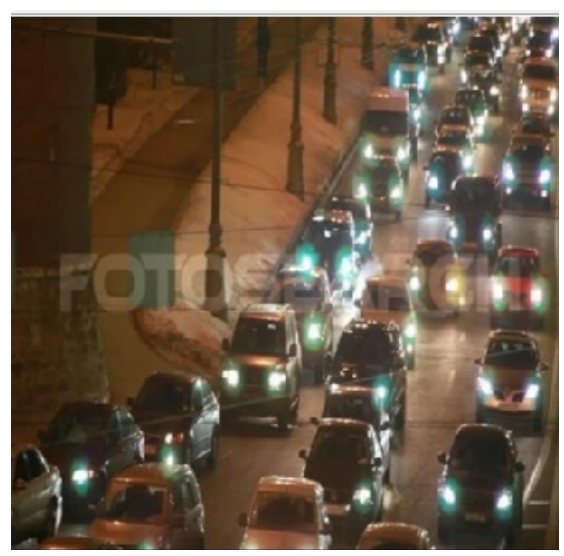

(d)

Fig(c) and(d) represents the input video and the preprocessed image.

\section{MODULE DESCRIPTION}

\section{A.LOAD VIDEO:}

The input video can be loaded and it is converted into frames by using $[\mathrm{X}]=\operatorname{aviread}(. .$. $\mathrm{A}=\operatorname{aviread}$ (pathname,filename) reads a grayscale or color image from the file specified by the string filename. If the file is not in the current folder, or in a folder on the MATLAB path, specify the full pathname. The input video is in avi format. Here are Converting into frames as rate as 15 frames paer second.The video which we used here is a car traffic video.For our video we can get (20 X 10) frames,Totally we can get 200 frames.

\section{B.PREPROCESSING:}

Preprocessing is nothing but removal of noise from frames. There are several filters to remove the noise, in particular we are using the Gaussian filter. The noise may be due to displacement of lens in our camera, or while transformation of an image.As shown in fig(d). The Gaussian filter is a non-uniform low pass filter. Generally noise may get included due to change in atmospheric condition.It is important to remove noise to make sure that vehicle's headlights are very clear.

\section{C.EDGE BASED DETECTION:}

Edge detection is the approach used most frequently for segmenting images based on the abrupt(local) changes in intensity. Edge detection is used to detect and link edge pixels to form contours. There are several steps involved in edge detection they are smoothing, enhancement, detection and localization. In this paper the edges are detected based on gradient edge detection 
International Journal of Information Sciences and Techniques (IJIST) Vol.4, No.3, May 2014

because laplacian edge detection are fail to detect the edge with high intensity point variations and also it is impossible to detect the image which has so much of noise.It is noted that there are several detectors in gradient edge detection.Here we are using canny edge detector to detect the edges in congested scenes

\section{D.BRIGHT OBJECT EXTRACTION:}

For extracting the headlights here we are using the thresholding technique. We know that in the night time, headlights pixels will have a high intensity value. Based on that we can segment the headlight. Suppose that the gray-level histogram corresponds to an image, $f(x, y)$, composed of dark objects in a light background, in such a way that object and background pixels have gray levels grouped into two dominant modes. One obvious way to extract the objects from the background is to select a threshold ' $\mathrm{T}$ ' that separates these modes. Then any point $(\mathrm{x}, \mathrm{y})$ for which $f(x, y)>T$ is called an object point, otherwise, the point is called a background point.

As a result we can get the bright objects which consists of vehicle's headlight and also other bright objects.

\section{E.MORPHOLOGICAL OPERATION}

Even though the bright objects are extracted it is important to extract the headlights of a vehicle separately. We know that in night time road reflection cause a major problem. Hence it is important to remove those reflection, for that here we are using the morphological operation. It is noted that some these reflection may reduce the accuracy of counting. That why it is important to suppress the road reflection. There are several types of morphological operators in particular we are using opening and closing operator.As a result we can get only the headlight of the vehicle.

\section{F.VEHICLE TRACKING AND PAIRING:}

From the morphological operation we could get the binary image. For tracking and pairing the vehicle headlight we are using bidirectional algorithm. The algorithm works in the following way. According to the intensity value, the pixels are grouped. If the grouped pixels are of similar size then it is consider as two headlights are belongs to the same vehicle. Thus the headlights are detected from the vehicle. From the grouped pixels centroid point is calculated. If any of the centroid point are lie on the same line then the two headlights are paired as a result vehicle count has been increamented. vehicle tracking and identification process includes three phases. First, the vehicle component tracking process is associated with the motion relation of vehicle components in succeeding frames by analyzing their spatial and temporal features. Then, the phase of motion-based grouping process is applied to the tracked vehicle components to construct whole moving vehicles. These moving vehicles are then tracked in the vehicle tracking phase. Finally, the vehicle recognition phase identifies and classifies the types of tracked vehicles. 


\section{V.EXPERIMENTAL RESULT}

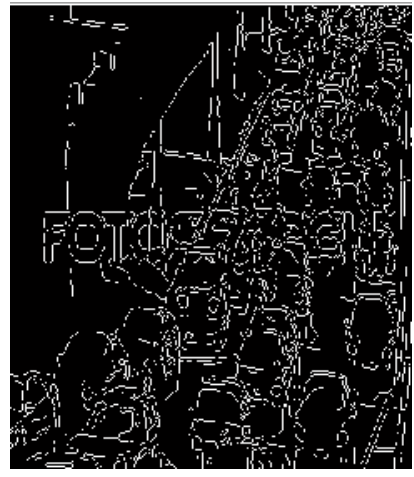

(e)

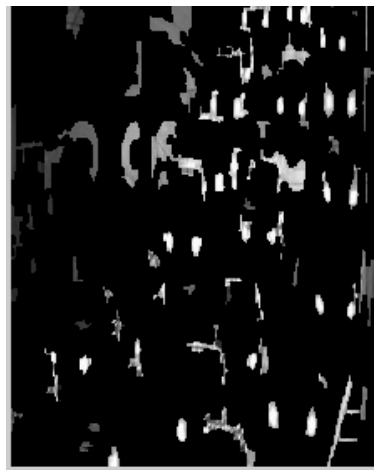

(f)

The Fig(e) represents the edge detection output and fig(f) represents the bright light extraction

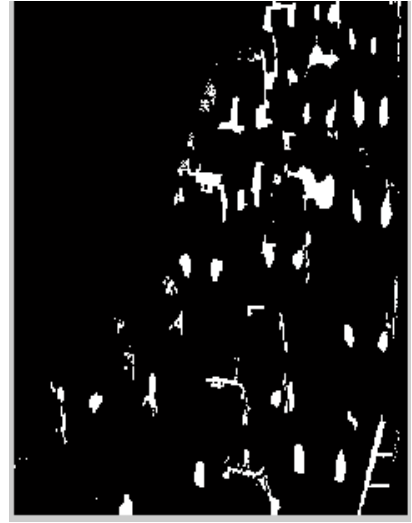

(g)

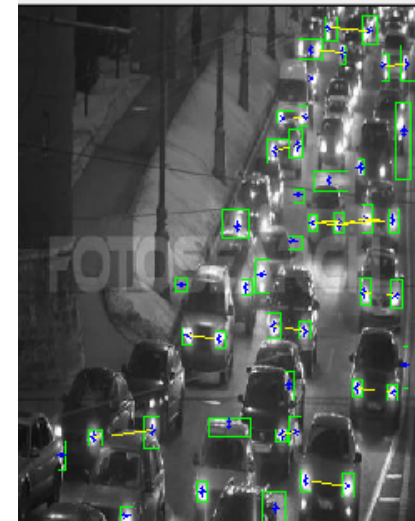

(h)

The fig ( $\mathrm{g}$ ) represents the output of morphological operation and fig $(\mathrm{h})$ represents the pairing of vehicle's headlights

\section{A RESULTS ON EDGE DETECTION}

Above fig (e) reprsents the output of edge detection.Canny edge detector is used to detect the edges in congested scenes, because it has good detection,good localization and minimal response.Canny edge detector uses multi-stage algorithm to detect a wide range of edges in an image.Those stages are noise reduction,finding the intensity gradient of the image non maximum suppression and tracing edges through the image and hysteresis thresholding

\section{B RESULTS ON HEADLIGHT EXTRACTION}

In nighttime video scenes to represent the presence ofvehicles headlight is a strong and consistent feature.For that purpose by applying the thresholding method, the headlights of movingvehicles can be efficiently segmented in nighttime traffic images as shown in fig 
International Journal of Information Sciences and Techniques (IJIST) Vol.4, No.3, May 2014

(f).Here we are setting the 240 pixels as the threshold value. According to that pixel value, it segment the pixels of headlights in traffic image sequences.

\section{RESULTS ON THRESHOLDING}

Above fig(f) represents the bright light extraction,to count the number of vehicles in night scenes ,the headlight is a strong and consistent feature In this study, the pixels of the headlight images are segmented in grayscale images by the thresholding method. Here the threshold value is set as 240 pixels.

\section{RESULTS ON MORPHOLOGICAL OPERATION}

After segementing the headlight portions, but still there is some reflections which are mainly due to the common light sources, such as rear lights, street lamps and some reflections of headlights .These Reflections are the main source of interference with headlight detection. We can differentiate between headlights and reflections by their shapes because the shape of headlights were stable when compare to their reflections. Therefore by using morphological operation we can suppress the reflections on the road surface as shown in the fig.

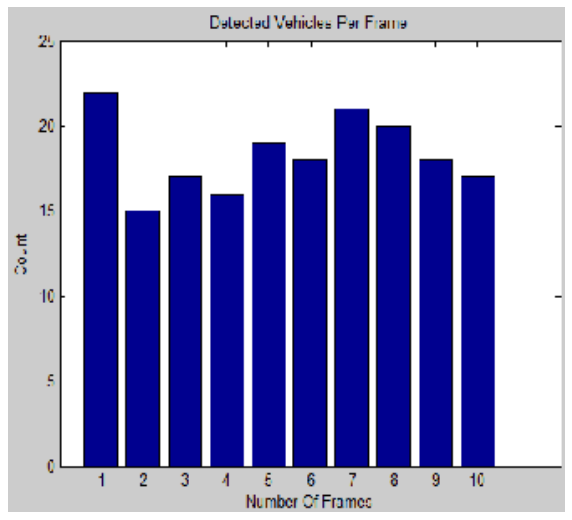

Fig(j) graph has been plotted between no. of frames and count

\begin{tabular}{|l|l|l|l|}
\hline S.NO & $\begin{array}{l}\text { NUMBER } \\
\text { OF } \\
\text { VEHICLES }\end{array}$ & $\begin{array}{l}\text { DETECTION } \\
\text { TIME }\end{array}$ & ACCURACY \\
\hline 1 & 22 & 0.5860 & $95.5 \%$ \\
\hline 2 & 15 & 0.4054 & $96.3 \%$ \\
\hline 3 & 17 & 0.3665 & $97.5 \%$ \\
\hline 4 & 16 & 0.3554 & $98.2 \%$ \\
\hline
\end{tabular}

Fig(i) tabulation represents the number vehicles detected per frame

\section{CONCLUSION}

The proposed method has produced the vehicle detection and tracking systems in night time for identifying and classifying the moving vehicles in congested area. Intially the edges of the objects are detected by using a canny edge detector. The bright-object extraction in the segmentation process done by the thresholding technique. Then the headlights of the vehicles has tracked by using the connected component labeling technique and that headlights can be paired by the bidirectional reasoning algorithm. From this we can get the number of vehicles accurately without any fault. 
International Journal of Information Sciences and Techniques (IJIST) Vol.4, No.3, May 2014

\section{FUTURE ENHANCEMENT}

For further studies, the vehicle type classification function can be further improved and extended by integrating some sophisticated machine learning techniques such as support vector machine classifiers on multiple features, including vehicle lights and vehicle bodies, to further enhance the classification capability on more detailed vehicle types, such as sedans, buses, trucks, lorries, and

light and heavy motorbikes therefore by using this result we can separate four wheelers and two wheelers.

\section{REFERENCES}

[1] S. Tsugawa, "Vision-based vehicles in Japan: Machine vision systems and driving control systems," IEEE Trans. Ind. Electron., vol. 41, no. 4, pp. 398-405, Aug. 1994.

[2] Z. Sun, G. Bebis, and R. Miller, "On-road vehicle detection: A review," IEEE Trans. Pattern Anal. Mach. Intell., vol. 28, no. 5, pp. 694-711, May 2006.

[3] Broggi, M. Bertozzi, A. Fascioli, and G. Conte, Automatic Vehicle Guidance: The Experience of the ARGO Autonomous Vehicle. Singapore: World Scientific, 1999.

[4] H. Mori, M. Charkari, and T. Matsushita, "On-line vehicle and pedestrian detections based on sign pattern,” IEEE Trans. Ind. Electron., vol. 41, no. 4, pp. 384-391, Aug. 1994.

[5] A. Broggi, M. Cellario, P. Lombardi, and M. Porta, "An evolutionary approach to visual sensing for vehicle navigation," IEEE Trans. Ind. Electron., vol. 50, no. 1, pp. 18-29, Feb. 2003.

[6] T. Bücher, C. Curio, J. Edelbrunner, C. Igel, D. Kastrup, I. Leefken, G. Lorenz, A. Steinhage, and W. Seelen, "Image processing and behavior planning for intelligent vehicles," IEEE Trans. Ind. Electron., vol. 50, no. 1, pp. 62-75, Feb. 2003.

[7] S. Gupte, O. Masoud, R. F. K. Martin, and N. P. Papanikolopoulos, "Detection and classification of vehicles," IEEE Trans. Intell. Transp. Syst., vol. 3, no. 1, pp. 37-47, Mar. 2002.

[8] B.-F. Wu, S.-P. Lin, and Y.-H. Chen, "A real-time multiple-vehicle detection and tracking system with prior occlusion detection and resolution," in Proc. IEEE Int. Symp. Signal Process. Inf. Technol., Dec. 2005, pp. 311-316.

[9] J. Zhou, D. Gao, and D. Zhang, "Moving vehicle detection for automatic traffic monitoring," IEEE Trans. Veh. Technol., vol. 56, no. 1, pp. 51-59, Jan. 2007.

[10] W. F. Gardner and D. T. Lawton, "Interactive model-based vehicle tracking," IEEE Trans. Pattern Anal. Mach. Intell., vol. 18, no. 11, pp. 1115-1121, Nov. 1996.

[11] G. D. Sullivan, K. D. Baker, A. D. Worrall, C. I. Attwood, and P. M. Remagnino, "Model-based vehicle detection and classification using orthographic approximations," Image Vis. Comput., vol. 15, no. 8, pp. 649-654, Aug. 1997.

[12] D. Koller, J. Weber, T. Huang, J. Malik, G. Ogasawara, B. Rao, and S. Russell, “Towards robust automatic traffic scene analysis in real-time," in Proc. Int. Conf. Pattern Recog., 1994, vol. 1, pp. $126-131$.

[13] N. Peterfreund, "Robust tracking of position and velocity with Kalman snakes," IEEE Trans. Pattern Anal. Mach. Intell., vol. 21, no. 6, pp. 564- 569, Jun. 1999.

[14] S.-T. Tseng and K.-T. Song, "Real-time image tracking for traffic monitoring," in Proc. IEEE 5th Int. Conf. Intell. Transp. Syst., 2002, pp. 10-14.

[15] L.-W. Tsai, J.-W. Hsieh, and K.-C. Fan, "Vehicle detection using normalized color and edge map," IEEE Trans. Image Process., vol. 16, no. 3, pp. 850-864, Mar. 2007.

[16] D. Beymer, P. McLauchlan, B. Coifman, and J. Malik, "A real-time computer vision system for measuring traffic parameters,” in Proc. IEEE Conf. Comput. Vis. Pattern Recog., Jun. 1997, pp. 495510. 